\title{
Atypical presentation of rotational vertebral artery insufficiency: illustrative case
}

\author{
Pranish A. Kantak, MPH, ${ }^{1}$ Sarv Priya, MBBS,${ }^{2}$ Girish Bathla, MBBS, ${ }^{2}$ Mario Zanaty, MD, ${ }^{1}$ and Patrick W. Hitchon, $M{ }^{1}$ \\ 1Department of Neurological Surgery and 2Division of Neuroradiology, Department of Radiology, University of lowa Hospitals and Clinics, lowa City, lowa
}

\begin{abstract}
BACKGROUND Rotational vertebral artery insufficiency (RVAl), also known as bow hunter's syndrome, is an uncommon cause of vertebrobasilar insufficiency that leads to signs of posterior circulation ischemia during head rotation. RVAl can be subdivided on the basis of the anatomical location of vertebral artery compression into atlantoaxial RVAI (pathology at C1-C2) or subaxial RVAI (pathology below C2). Typically, RVAl is only seen with contralateral vertebral artery pathologies, such as atherosclerosis, hypoplasia, or morphological atypia.

OBSERVATIONS The authors present a unique case of atlantoaxial RVAI due to rotational instability, causing marked subluxation of the C1-C2 facet joints. This case is unique in both the mechanism of compression and the lack of contralateral vertebral artery pathology. The patient was successfully treated with posterior C1-C2 instrumentation and fusion.

LESSONS When evaluating patients for RVAl, neurosurgeons should be aware of the variety of pathological causes, including rotational instability from facet joint subluxation. Due to the heterogeneous nature of the pathologies causing RVAl, care must be taken to decide if conservative management or surgical correction is the right course of action. Because of this heterogeneous nature, there is no set guideline for the treatment or management of RVAl.
\end{abstract}

https://thejns.org/doi/abs/10.3171/CASE20169

KEYWORDS bow hunter's syndrome; bow hunter's stroke; rotational vertebral artery occlusion; rotational vertebral artery insufficiency; RVAl; RVAO; BHS

Rotational vertebral artery insufficiency (RVAl), also known as bow hunter's syndrome or bow hunter's stroke, is an uncommon cause of vertebrobasilar insufficiency (VBI) that leads to signs of posterior circulation ischemia during head rotation, such as syncope, dizziness, visual field defects, and vertigo. This syndrome was first described in 1978 by Sorensen, ${ }^{1}$ who described a patient who developed Wallenberg syndrome secondary to posterior infarction during archery practice. ${ }^{1-5}$ Typically, RVAl occurs in male patients aged 50-79 years, with hypertension, osteoarthritis, hyperlipidemia, diabetes, smoking, and coronary artery disease all considered important risk factors. ${ }^{6}$

Because of the long course of the vertebral artery (VA), compression can occur at any level and is divided into atlantoaxial RVAI (involving C1-C2) and subaxial RVAI (involving levels below C2). ${ }^{3}$ In most instances, unilateral compression alone is not sufficient for symptoms to occur. Most commonly, symptoms occur when patients have a contralateral hypoplastic VA or some variant in the normal course or caliber of the contralateral VA, such as severe atherosclerosis, VAs terminating in the posterior inferior cerebellar artery (PICA), or the presence of a dominant persistent first intersegmental artery. $2,3,5,7-9$

We present a unique case of atlantoaxial RVAI due to rotational instability, causing marked subluxation of the $\mathrm{C} 1-\mathrm{C} 2$ facet joints. This case is unique in both the mechanism of compression and the lack of contralateral VA pathology. The patient was successfully treated with posterior $\mathrm{C} 1-\mathrm{C} 2$ instrumentation and fusion.

\section{Illustrative Case}

\section{History and Preoperative Assessment}

A 37-year-old male presented to the neurosurgery clinic from his primary care provider because of 15-20 years of episodes of blurry vision, contraction of his visual field, lightheadedness, and headaches upon turning his head to the right side. He had never lost consciousness entirely, nor had he had any falls or significant trauma to his neck or head. He found that these symptoms were reproducible by turning his

ABBREVIATIONS $\mathrm{AI}=$ atlantoaxial instability; $\mathrm{CTA}=$ computed tomography angiogram; $\mathrm{PICA}=$ posterior inferior cerebellar artery; $\mathrm{RVAI}=$ rotational vertebral artery insufficiency; VA = vertebral artery; VBI = vertebrobasilar insufficiency.

INCLUDE WHEN CITING Published March 1, 2021; DOI: 10.3171/CASE20169.

SUBMITTED December 21, 2020. ACCEPTED January 13, 2021.

(C) 2021 The authors, CC BY-NC-ND 4.0 (http://creativecommons.org/licenses/by-nc-nd/4.0/). 
head to the right or bending down to pick objects off the floor and occurred several times each day. Occasionally, he experienced numbness and tingling in his hands during these episodes. Over the last year, these episodes had become more frequent and had led to the limitation in the range of motion of his head with external rotation to the right.

On examination, the patient did have some limitation in the range of motion of his head, particularly head turning to the right. There was no evidence of myelopathy or radiculopathy. His face was symmetrical, and the result of his cranial nerve examination was unremarkable. His primary care provider had ordered a dynamic computed tomography angiogram (CTA) with his head in a neutral (Fig. 1 left) and rightward externally rotated position (Fig. 1 right). The bilateral VAs were visualized and originated from the subclavian arteries without significant ostial stenosis and were otherwise within normal limits. The cervical and intracranial VAs were of normal course and caliber. Upon head rotation to the right, this CTA showed marked subluxation of the C1-C2 facet joints. The C1 lateral mass subluxated posteriorly on the right and anteriorly on the left. This subluxation resulted in occlusion of the V3 segment of the left VA and was deemed the cause of his symptomatology. This can also be seen in Fig. 2 as three-dimensional reformats of the computed tomography images with segmentation of the vertebral vasculature.

\section{Operative Course}

The patient was brought to the operating room and intubated under general anesthesia. His head was fixed in a 3-point fixation device, and he was turned prone onto the Mzuho Jackson table. Under fluoroscopy, a midline incision was made and extended to identify the spinous processes and laminae of $\mathrm{C} 1$ and $\mathrm{C} 2$. Bilateral $\mathrm{C} 2$ nerve roots were then sacrificed, exposing the $\mathrm{C} 1-\mathrm{C} 2$ facet joints bilaterally, which were separated without the restraints generally provided by the capsular ligaments. Under fluoroscopy, $3.5 \times 30-\mathrm{mm}$ screws were placed into the lateral masses of $\mathrm{C} 1$. Similarly, at C2, $3.5 \times 22-\mathrm{mm}$ screws were

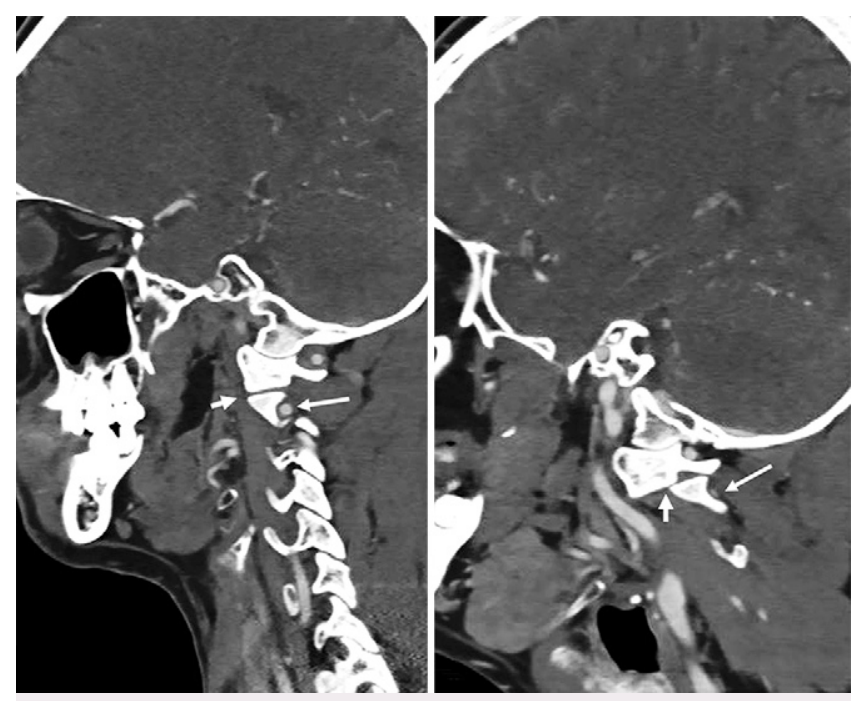

FIG. 1. Sagittal multiplanar reformations with the head in the neutral position (left) and rotated toward the right side (right). Normal articulation of the left C1-C2 joint (short arrow in left) with VA posterior to $\mathrm{C} 2$ vertebral body (long arrow in left) is noted in a neutral position. On rightward rotation, there is prominent subluxation at C1-C2 articulation (short arrow in right) with significant attenuation of the VA (long arrow in right).
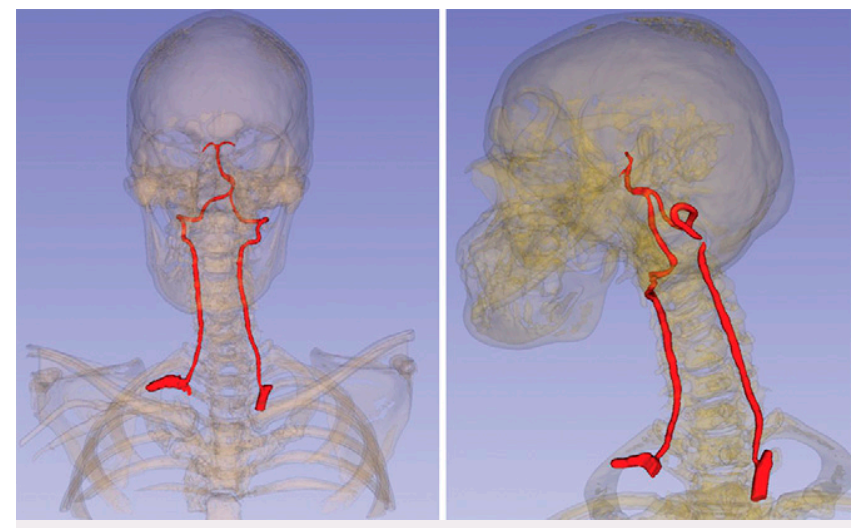

FIG. 2. Three-dimensional reformats of the computed tomography images with segmentation of the vertebral vasculature. The left VA is normal in a neutral position (left) but shows significant attenuation with the head rotated toward the right side (right).

placed into the pars of $\mathrm{C} 2$. Thirty-millimeter rods, $3.5 \mathrm{~mm}$ in diameter, spanned the $\mathrm{C} 1-\mathrm{C} 2$ screws on either side. The spacing between the rods and screw saddles was adjusted to provide for the posterior reduction of $\mathrm{C} 1$. Cortical cancellous bone was placed on the lamina between C1 and C2 after decorticating the $\mathrm{C} 1-\mathrm{C} 2$ lamina process. The patient tolerated the procedure well, was extubated, and was transferred to recovery without any acute complications.

\section{Postoperative Course}

During his 6-week follow-up visit, the patient reported improvement in his level of lightheadedness, headaches, and improvement in his visual field. However, he was still experiencing discomfort at the back of his head. Standing C-spine films were completed before his appointment and showed postsurgical changes of a posterior spinal fusion without hardware complications (Fig. 3). On examination, his incision appeared well healed without erythema or tenderness. He was instructed to normalize his activity and attend follow-up again in 3 months with repeated imaging.

\section{Discussion}

RVAl is a syndrome that is characterized by symptoms of $\mathrm{VBI}$, such as vertigo, nystagmus, and syncope, when the head is rotated. The first
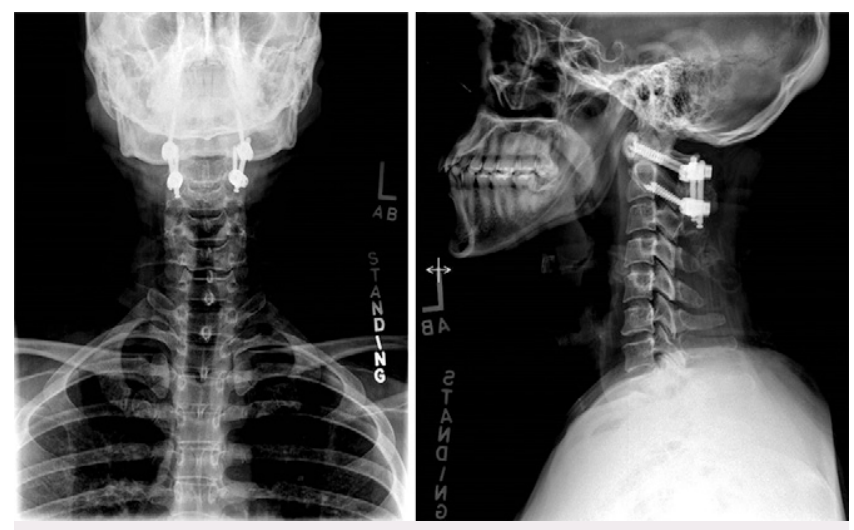

FIG. 3. Anteroposterior (left) and lateral (right) radiographs obtained 6 weeks after surgery, showing the hardware in place. 
description of VBI due to head rotation was described by de Kleyn and Versteegh in 1933;10 however, the syndrome of RVAI or bow hunter's syndrome was not described until 1978 by Sorensen. ${ }^{1}$ As mentioned above, Sorensen described a patient who developed symptoms similar to lateral medullary, or Wallenberg, syndrome after a lengthy archery practice. Wallenberg syndrome is characterized by swallowing difficulties, nystagmus, vertigo, and pain/temperature loss on the ipsilateral face and contralateral body due to PICA or VA branch occlusion. ${ }^{1,11}$

In this case report, we present a unique case of RVAI due to rotational instability at the $\mathrm{C} 1-\mathrm{C} 2$ level. When our patient turned his head to his right side, he would sublux at his $\mathrm{C} 1-\mathrm{C} 2$ facet joints, causing compression of his ipsilateral VA. Our patient had been experiencing symptoms for 15-20 years when his symptoms began to worsen and increase in frequency. No interventions had been performed up to this time. Interestingly, he did not have any anatomical variants or a malformed contralateral artery that could be the cause of his symptoms. Typically, adequate perfusion of the posterior circulation can be achieved through the contralateral vessel, and patients do not become symptomatic. Because his symptoms were worsening and an obvious, correctable source of his pathology was seen, we proceeded with a C1-C2 posterior fusion. At his 6-week follow-up, he had satisfactory improvement of his symptoms.

The VAs typically originate from the subclavian arteries bilaterally and are divided into 4 segments, termed V1-V4. V1, or the prevertebral part, travels superiorly and posteriorly between the longus colli and anterior scalene muscles. The V2, or cervical part, runs through the $\mathrm{C} 6-\mathrm{C} 2$ transverse foramina, anterior to the cervical nerve trunks. The V3, or atlantic part, begins at the $\mathrm{C} 2$ transverse foramen and can be subdivided into a vertical part, which passes upward over the $\mathrm{C} 2$ nerve root and enters the transverse foramen of $\mathrm{C} 1$, and a horizontal part, which curves medially and posteriorly behind the superior articular process of the atlas. The horizontal portion then lies in the groove on the upper surface of the posterior arch of the atlas. The V4, or intracranial part, pierces the dura mater and courses medially on the anterior surface of the medulla. $6.12,13$

In a 2015 review, Jost and Dailey ${ }^{14}$ detailed the anatomical variations of RVAI on the basis of 126 patients. Stenosis in the V1 distribution was the culprit in $4 \%$ of patients, with $58 \%$ in the V2 distribution and $36 \%$ in the V3 distribution, and only $2 \%$ showing pathology distal to C1. Rastogi et al. ${ }^{15}$ described the atlantoaxial RVAI (C1-C2) segment as being involved in 99 of 142 patients with bow hunter's syndrome. This discrepancy between the above 2 literature reviews is best attributed to inconsistent criteria involving numerous patients from different centers. ${ }^{14,15}$

In subaxial RVAI, the ipsilateral VA is often compressed by a bony spur of a hypertrophic uncovertebral joint, adjacent segment disease after anterior instrumentation, muscle bands, an extraosseous vertebral V2 segment, or a laterally herniated disc during contralateral head rotation. This is the most common subtype of RVAl reported in the literature. ${ }^{3,4,8,16-19}$ In atlantoaxial RVAI, the V3 portion of the VA is compressed and can also be occluded with rotational head movement, which is presented in this illustrative case. This is most commonly caused by an ossified or thickened atlanto-occipital membrane, degenerative changes from cervical spondylosis, a dural fold in the foramen magnum, an accessory ossicle behind the atlanto-odontoid junction, C1-C2 facet hypertrophy, muscular and tendinous insertions, erosive rheumatoid arthritis, or dynamic instability, as in this case. . $7,9,14,18,20,21$ At the $\mathrm{C} 1-\mathrm{C} 2$ transverse foramen, the V3 segment is relatively immobile, which may account for its susceptibility to rotational obstruction. ${ }^{22}$

In a nonpathological situation, during head movement, the ipsilateral lateral mass of the atlas is translated posteriorly along the articular facet of the axis. In contrast, the contralateral lateral mass of the atlas is translated anteriorly along the articular facet of the axis. Rotational movement is limited by the alar (anteriorly) and transverse (posteriorly) ligaments. ${ }^{23}$ In our patient, upon head rotation to the right, the left lateral mass was subluxated anteriorly beyond normal alignment, whereas the ipsilateral lateral mass subluxated posteriorly. This was deemed to be the cause of the compression at the transverse foramen of the axis, which resulted in a significant decrease in blood flow through the vessel.

Atlantoaxial instability $(\mathrm{Al})$ is classified into 3 categories based on inflammatory, congenital, and traumatic etiologies. These are briefly discussed further. Because there are many synovial joints and ligaments that contribute to the stability of the upper cervical spine, rheumatoid arthritis is one of the most prevalent causative agents that promotes the development of Al. Chronic inflammation leads to laxity and stretching of the alar and transverse ligaments, erosion of the bony structures, and subsequent subluxation and instability. Several congenital disorders can also lead to Al, with the most prevalent being Down syndrome, which can cause laxity in the ligaments and bony abnormalities. Finally, traumatic injuries typically result from head trauma, which disrupts the alar, apical, or transverse ligaments, leading to $\mathrm{Al}^{24}$ Interestingly, our patient did not have any apparent causes for his $\mathrm{Al}$; however, given his long history of RVAl symptoms, this could have been due to a remote head trauma history that went unnoticed.

As one might expect, management options for these patients are wide ranging, from conservative watchful management to surgical treatment. Currently, there are still no standard treatments for RVAI, given the rarity of the diagnosis. Conservative management includes avoiding head and neck rotation by using a neck brace or collar and antiplatelet or anticoagulation therapy. Few studies have looked at the outcomes of conservative management versus surgical treatment, partly because of the diverse pathologies that can cause RVAI. Choi et al. analyzed 21 patients with RVAl. ${ }^{9}$ Twelve patients had "typical" RVAl, defined as compression between $\mathrm{C} 1$ and $\mathrm{C} 2$ with either contralateral VA hypoplasia or occlusion. Of these 12 patients, 2 proceeded to surgery with a $\mathrm{C} 1-\mathrm{C} 2$ fusion, and the other 10 patients were managed conservatively. Of the conservatively managed patients, only 1 had an adverse outcome of middle cerebral stroke not related to RVAI. The other 9 patients were defined as "atypical" RVAl and had different compression patterns, such as compression of bilateral VAs or compression at different cervical levels. Three of these patients had a normal contralateral VA. These patients were all managed conservatively and had no adverse events. In contrast with these patients, our patient would have been classified as a mix between typical and atypical RVAI because his compression was at a traditional level but without contralateral VA pathology. These results suggest that conservative management may be an option for patients with RVAI, depending on their level of compression. Rastogi and colleagues, ${ }^{15}$ in their 2015 review of 153 cases, showed a large diversity of treatment modalities as well. A surgical approach was seen in 105 of 146 cases, with 39 patients treated conservatively and 2 patients treated with a combination of conservative and surgical management ( $p<0.0001) .8,9,15$ With regard to our patient, because his symptoms had been accelerating over the past year and his CTA showed substantial evidence of rotary subluxation at C1-C2, a surgical approach was deemed necessary to control his symptoms.

\section{Observations}

In this case report, we report a unique case of rotational VA insufficiency due to subluxation of the articular facet at C1-C2 when our 
patient turned his head to the right. This case was a result of an old injury. When our patient turned his head to the right, the subluxation caused compression of the V3 segment of the VA in the transverse foramen of the axis. Furthermore, our patient presented without contralateral VA pathology, further adding to the atypical nature of his symptoms.

\section{Lessons}

When evaluating patients for RVAl, neurosurgeons should be aware of the variety of pathological causes, including rotational instability from facet joint subluxation. Given the heterogeneous nature of the pathologies causing RVAl, care must be taken to decide if conservative management or surgical correction is the right course of action. Because of this heterogeneous nature, there is no set guideline for the treatment or management of RVAI. In the setting of C1-C2 rotatory subluxation; however, $\mathrm{C} 1-\mathrm{C} 2$ instrumentation and fusion are recommended.

\section{Acknowledgments}

We thank the patient presented in this case. Patients indeed are our most significant teachers.

Dr. Bathla received grants from the American Cancer Society and the Foundation for Sarcoidosis Research during the conduct of this study.

\section{References}

1. Sorensen BF. Bow hunter's stroke. Neurosurgery. 1978;2(3): 259-261.

2. Buch VP, Madsen PJ, Vaughan KA, et al. Rotational vertebrobasilar insufficiency due to compression of a persistent first intersegmental vertebral artery variant: case report. J Neurosurg Spine. 2017;26(2):199-202.

3. Luzzi S, Gragnaniello C, Marasco S, et al. Subaxial vertebral artery rotational occlusion syndrome: an overview of clinical aspects, diagnostic work-up, and surgical management. Asian Spine J. Published online September 10, 2020. doi:10.31616/ asj.2020.0275

4. Duan G, Xu J, Shi J, Cao Y. Advances in the pathogenesis, diagnosis and treatment of bow hunter's syndrome: a comprehensive review of the literature. Intervent Neurol. 2016;5(1-2): 29-38.

5. Go G, Hwang S-H, Park IS, Park H. Rotational vertebral artery compression: bow hunter's syndrome. J Korean Neurosurg Soc. 2013;54(3):243-245.

6. Davis DD, Kane SM. Rotation vertebral artery syndrome. In: StatPearls. Treasure Island, FL: StatPearls Publishing; 2020. Accessed November 15, 2020. https://www.ncbi.nlm.nih.gov/ books/NBK559022/

7. Yang PJ, Latack JT, Gabrielsen TO, et al. Rotational vertebral artery occlusion at C1-C2. AJNR Am J Neuroradiol. 1985;6(1): $96-100$.

8. Lu DC, Zador Z, Mummaneni PV, Lawton MT. Rotational vertebral artery occlusion-series of 9 cases. Neurosurgery. 2010;67(4): 1066-1072.

9. Choi KD, Choi JH, Kim JS, et al. Rotational vertebral artery occlusion: mechanisms and long-term outcome. Stroke. 2013; 44(7):1817-1824.

10. de Kleyn A, Versteegh C. Über verschiedene Formen von Ménières Syndrom. Dtsch Z Nervenheilkd. 1933;132:157-189.

11. Wallenberg syndrome. Genetic and Rare Diseases Information Center. Accessed December 2, 2020. https://rarediseases.info.nih. gov/diseases/9263/wallenberg-syndrome
12. Weerakkody Y, Gaillard F. Vertebral artery. Radiopaedia. Accessed December 2, 2020. https://radiopaedia.org/articles/ vertebral-artery?lang=us

13. Vertebral artery. IMAIOS. Accessed December 2, 2020. https:// www.imaios.com/en/e-Anatomy/Anatomical-Parts/Vertebral-artery

14. Jost GF, Dailey AT. Bow hunter's syndrome revisited: 2 new cases and literature review of 124 cases. Neurosurg Focus. 2015;38(4):E7.

15. Rastogi $\mathrm{V}$, Rawls A, Moore O, et al. Rare etiology of bow hunter's syndrome and systematic review of literature. J Vasc Interv Neurol. 2015;8(3):7-16.

16. Buchanan CC, McLaughlin N, Lu DC, Martin NA. Rotational vertebral artery occlusion secondary to adjacent-level degeneration following anterior cervical discectomy and fusion. J Neurosurg Spine. 2014;20(6):714-721.

17. Rendon R, Mannoia K, Reiman S, et al. Rotational vertebral artery occlusion secondary to completely extraosseous vertebral artery. J Vasc Surg Cases Innov Tech. 2018;5(1):14-17.

18. Kuether TA, Nesbit GM, Clark WM, Barnwell SL. Rotational vertebral artery occlusion: a mechanism of vertebrobasilar insufficiency. Neurosurgery. 1997;41(2):427-433.

19. Sumdani H, Shahbuddin Z, Church P. Rotational vertebrobasilar insufficiency provoked by distorted trajectory of subclavian artery. J Vasc Med Surg. 2019;7(2):381.

20. Trivedi T, Reddi R, Kumar A, Agarwal B. Recurrent vertebrobasilar insufficiency-bow hunter's syndrome. J Emerg Crit Care Med. 2018;2:65

21. Whitmore RG, Simon SL, Hurst RW, et al. Bow hunter's syndrome caused by accessory cervical ossification: posterolateral decompression and the use of intraoperative Doppler ultrasonography. Surg Neurol. 2007;67(2):169-171.

22. Barton JW, Margolis MT. Rotational obstructions of the vertebral artery at the atlantoaxial joint. Neuroradiology. 1975;9(3):117-120.

23. Bogduk N. Functional anatomy of the spine. Handb Clin Neurol. 2016;136:675-688.

24. Lacy J, Bajaj J, Gillis C. Atlantoaxial instability. In: StatPearls. Treasure Island, FL: StatPearls Publishing; 2020. Accessed October 5, 2020. https://www.ncbi.nlm.nih.gov/books/NBK519563/

25. Mapstone T, Spetzler RF. Vertebrobasilar insufficiency secondary to vertebral artery occlusion from a fibrous band. Case report. J Neurosurg. 1982;56(4):581-583.

\section{Disclosures}

Dr. Bathla reported receiving grants from Siemens Healthineers and other from Bayer during the conduct of the study. No other disclosures were reported.

\section{Author Contributions}

Conception and design: Hitchon, Kantak, Zanaty. Acquisition of data: Kantak, Priya, Bathla. Analysis and interpretation of data: Priya, Bathla. Drafting the article: Hitchon, Kantak. Critically revising the article: Kantak, Priya, Bathla, Zanaty. Reviewed submitted version of manuscript: Hitchon, Kantak, Bathla. Administrative/technical/material support Bathla. Study supervision: Hitchon.

\section{Correspondence}

Patrick W. Hitchon: University of lowa Hospitals and Clinics, lowa City, IA. patrick-hitchon@uiowa.edu. 